
PATIENTS TREATED WITH PIRFENIDONE

doi:10.1136/thoraxjnl-2012-202678.419

R Frank, B Amies, J Capps, A Duck, CT Leonard, J Holme. University Hospital of South Manchester NHS Foundation Trust, Manchester, United Kingdom

Introduction and Objectives Idiopathic pulmonary fibrosis (IPF) is associated with a reduced life expectancy of $2-3$ years from diagnosis to death. In the CAPACITY ${ }^{1}$ study patients treated with pirfenidone had a slower decline in FVC (evident from 6 months), and showed a trend towards improved mortality. We investigated causes of death and factors influencing mortality in patients who have received pirfenidone on a Named Patient Programme.

Method We retrospectively analysed the data from 25 patients with a minimum of 3 months follow-up who were treated with Pirfenidone between August 2011 and June 2012 in a tertiary ILD clinic. Results Mean age was $67 \pm 8$ years and $19(76 \%)$ were male. 8 of the 25 patients treated died. 4 had discontinued pirfenidone prior to death. 4 died due to an IPF exacerbation, 3 due to progressive disease and 1 due to a combination of progressive disease and congestive cardiac failure. Survivors had a greater DLCO \% predicted at baseline $(49 \pm 16$ v $30 \pm 8 ; p=0.001)$ and were heavier $(82.8 \pm 14.6 \mathrm{~kg} \mathrm{v}$ $71.0 \pm 11.2 \mathrm{~kg} ; \mathrm{p}=0.032)$. A greater proportion of subjects who died $(75 \%)$ had severe IPF (DLCO $<35 \%$ predicted or $\mathrm{FVC}<50 \%$ predicted) compared with those who survived $(18 \%$; $p=0.014)$. For survivors compared with those who had died, there was a trend towards greater BMI $(28.7 \pm 4.4 \vee 25.4 \pm 3.9 ; \mathrm{p}=0.065)$ and younger age $(65 \pm 8.2 \vee 70 \pm 4.5 ; p=0.053)$.

FVC \% predicted, Chronic Respiratory Questionnaire Scores and the presence of significant co-morbidities at baseline did not predict survival.

Conclusion In IPF patients who had been treated with pirfenidone, all died of IPF related causes, and those who died weighed less, and had more severe disease with a lower baseline DLCO\% predicted.

This may help to inform us about the most appropriate patients for whom pirfenidone treatment should be considered.

1. Noble P, Albera C, Bradford W et al, for the CAPACITY Study Group. Pirfenidone in patients with idiopathic pulmonary fibrosis (CAPACITY): two randomised trials. Lancet 2011; 377: 1760-69.

\section{P137 PRACTISE AND EFFECTS OF AUTOIMMUNE SCREENING IN PULMONARY FIBROSIS}

doi:10.1136/thoraxinl-2012-202678.420

L Anning, MA Gibbons. Royal Devon \& Exeter NHS Foundation Trust, Exeter, United Kingdom

Introduction and Objectives Patients with pulmonary fibrosis can have features of autoimmune disorders that do not meet criteria for connective tissue disease (CTD), sometimes referred to as autoimmune-featured interstitial lung disease (AIF-ILD). Incorrect diagnosis and labelling could have important implications for treatment and prognosis.

We analysed the prevalence and type of immune profile performed to investigate patients with pulmonary fibrosis to determine what effect it had on subsequent management, and to assess variations in clinical practise.

Methods Retrospective review of patients attending the ILD and general respiratory clinics at our centre $(n=75)$ with a diagnosis of pulmonary fibrosis, not known to be due to a CTD, or to be caused by a drug reaction, hypersensitivity, or sarcoidosis.

Results Seventy-five cases were reviewed (76\% male). Age range was 55-88 years (median 73 years). Eighty percent of patients $(n=60)$ had immune tests performed, with 13\% $(n=10)$ having two tests, $5 \%(n=4)$ having three, and $61 \%(n=46)$ having four or more. Twenty-eight percent $(n=21)$ had a positive immune test. A positive immune screen partly directed the introduction of antiinflammatory therapy in $24 \%$ of patients $(n=5)$. One of these patients had a lung biopsy. In $38 \%$ of patients with a positive immune screen $(n=7)$ it was unclear if the result contributed to clinical decision making. Lung biopsies were performed in 19\% of patients with a positive immune screen, whereas only $8 \%$ of the total had a lung biopsy.

Conclusions These results show there is considerable variability in immune screen testing: which tests are performed, how positive tests are interpreted and whether they affect management. Our data hint at the usefulness of immune profiling, particularly where a lung biopsy has not been undertaken, and suggest that an abnormal immune profile increases the rate of lung biopsy. We suggest that a multi-centre, prospective, longitudinal study is required to further define AIF-ILD in terms of incidence, natural history, response to therapy and outcome. Whether AIF-ILD and the other idiopathic interstitial pneumonias are truly distinct remains to be determined, but clearly there is a need for advanced definitions, diagnostic algorithms, and management strategies.

\section{Studies in PAH and pulmonary thromboembolism}

\section{P138 BASELINE HYPOXEMIA PREDICTS SURVIVAL IN PATIENTS WITH IDIOPATHIC PULMONARY ARTERIAL HYPERTENSION WITHOUT PATENT FORAMEN OVALE}

doi:10.1136/thoraxjnl-2012-202678.421

A Nair, J Fuller, R Crackett, M Day, J Lordan, A Fisher, P Corris. Northern National Pulmonary Vascular Treatment Centre, Freeman Hospital, Newcastle upon Tyne, United Kingdom

Introduction and Objectives The relevance of hypoxemia in patients with Idiopathic pulmonary arterial hypertension (IPAH) without Patent Foramen Ovale (PFO) on haemodynamics and survival is not well described. The aim of this study is to ascertain the severity and prevalence of hypoxemia in patients with IPAH without PFO, and its impact on survival.

Methods This is a single centre registry evaluation from 2001-2010 of patients attending the National Pulmonary Vascular Treatment Centre for Northern England. Outcomes were prospectively recorded on database following comprehensive assessment and confirmation with right heart catheterisation. PFO was excluded using bubble contrast ECHO. Data on arterial blood gas (ABG), lung function and radiology were obtained from case notes/hospital reporting systems. Patients initiated on targeted therapy were followed up at 3-4 month intervals.

Results ABG data was available for a cohort of 44 IPAH patients. $28(58 \%)$ were women. Mean age (SD) was $55.6(16.6)$ years, mean (SE) for Mean PAP 47(1.7) $\mathrm{mm} \mathrm{Hg}$, CI 2.18(0.09) $\mathrm{L} / \mathrm{min} / \mathrm{m}^{2}$, 6MWD 266(22) metres, $\mathrm{PaO}_{2}$ 9.04(0.25) kPa, $\mathrm{SPO}_{2} \%$ 93(0.3), FEV1\% 81.1(2.8), DLCO\% 61.4(3.4). Mild Hypoxemia was seen in $7(16 \%)$, Moderate Hypoxemia in 26(59\%), Severe Hypoxemia in $11(25 \%)$ patients respectively. There were significant differences in survival between groups (logrank P value 0.0012, Figure 1). Mean survival (95\% CI) in mild, moderate and severe hypoxemia groups were 12.3 years (9.1 to 15.4 ), 6.84(4.8 to 8.8), and 2.8(1.6 to 3.9) years respectively. There were no correlations between $\mathrm{PaO} 2$ and haemodynamic measurements (RAP/CI/PVR)/FEV1/FC. A modest correlation r. 32 was observed between $\mathrm{PaO} 2$ and $6 \mathrm{MWD}$, (P0.04)

Conclusion Baseline Hypoxemia is an independent predictor of survival whilst on targeted therapy and moderate hypoxemia is prevalent in the absence of PFO/lung disease in IPAH. 


\section{Survival of IPAH patients without PFO sub groups with mild vs moderate vs severe hypoxemia:Survival proportions}

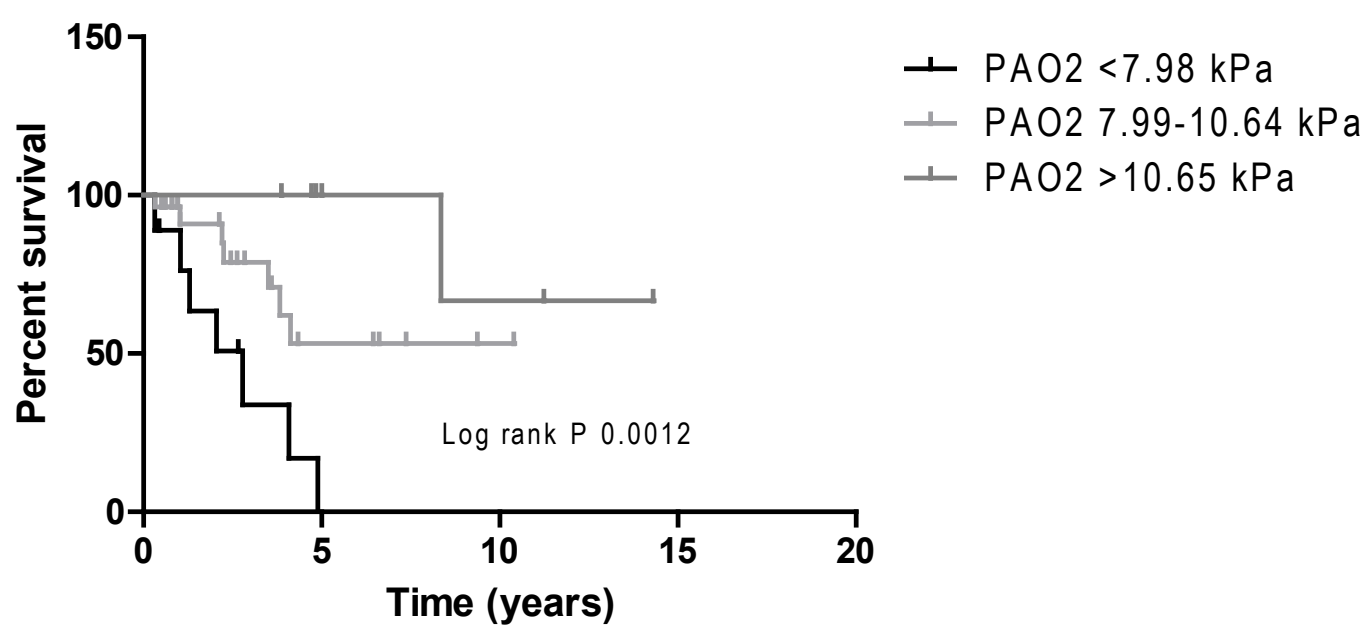

Abstract P138 Figure 1 Survival of IPAH patients without PFO based on severity of hypoxemia. Log rank comparisons HYPERTENSION BMPR2 MUTATION CARRIERS VS BMPR2 NON CARRIERS

doi:10.1136/thoraxjnl-2012-202678.422

CM Treacy, D Taboada Buasso, N Doughty, NW Morrell, J Pepke-Zaba. Papworth Hospital NHS Trust, Camrbidge, England

Introduction Idiopathic pulmonary arterial hypertension (IPAH) is a devastating condition charactericed by the narrowing and obliteration of small pulmonary arteries, leading to elevated pulmonary arterial pressure and ultimately to right heart failure and death. Even with treatment, IPAH survival is poor with 3 year survival reported to be $63 \%$ on epoprostenol (Mclaughlin, Circulation 2002). Pulmonary arterial hypertension (PAH) can be heritable, with mutations found in the bone morphogenetic protein type 2 receptor (BMPR2) gene found in $>70 \%$ of familial cases, (Lane, Nat Genet 2000). There are previous conflicting reports of worse survival in heritable PAH, but this has not previously been determined in the UK patient population.

Methods This study examined the retrospective database of 76 IPAH cases diagnosed between 2001-2006. 32 patients were screened for BMPR2 mutation. 13 were BMPR2+ve. Non-screened (NS) IPAH were included in the survival analysis $(n=44)$. Survival time was taken from the time of initial diagnostic right heart catherisation. Demographics, treatment, 6MWT, time to transplant and World Health Organization (WHO) classification were compared. Physicians were blinded to the BMPR2 status at time of treatment. Results All three cohorts presented at baseline with no significant difference in functional class, 6MWT, hemodynamics except age, cardiac index (CI), right atrial pressure (RAP) and treatment modalities. Patients with BMPR2 mutation were significantly younger. Of the $\mathrm{BMPR}+$ ve group, 6 patients were transplanted compared to zero in the BMPR-ve group. The NS IPAH cohort had 2 patients transplanted and fewer patients were treated with prostanoids. Time to transplant was shorter in the BMPR2 mutation carriers, 2.65 years, vs. 3.1years in the NS IPAH group.

Conclusion Survival for the first 5 years from diagnosis was similar in IPAH and heritable PAH. The BMPR2 + ve patients presented younger with severe disease and were treated more aggressively with 6 patients undergoing transplant in the 5 year period. BMPR2 mutation frequency was $41 \%$ from our cohort of 33 which is higher than previously reports of 20-30\% (Morrell, Proc Am Thorac Soc, 2006). Survival for IPAH continues to be poor even with improvements in treatment. Patients with BMPR2 mutations present with severe disease earlier and time to lung transplant is shorter.

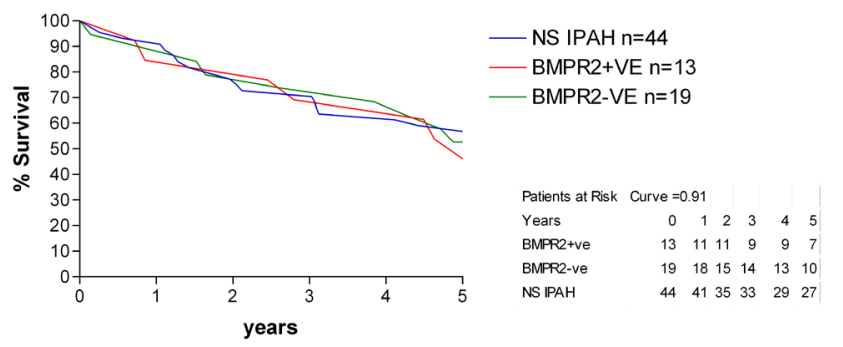

Abstract P139 Figure 1

P140 HIGH-DOSE OESTROGENS COMMENCED FOR GENDER
REASSIGNMENT SURGERY MAY PREDISPOSE TO
THE DEVELOPMENT OF CHRONIC THROMBOEMBOLIC
PULMONARY VASCULAR DISEASE

doi:10.1136/thoraxjnl-2012-202678.423

OLI Davies, CT McCabe, JW Pepke-Zaba, KK Sheares. Papworth Hospital, Cambridge, United Kingdom

Background Chronic thromboembolic pulmonary hypertension (CTEPH) often occurs as a sequela of venous thromboembolism (VTE) however, there appears to be marked differences in laboratory risk-profiles between CTEPH and VTE. Clinically, exogenous oestrogens (EO) are known to increase the risk of acute DVT in a dosedependent manner ${ }^{2}$ but a pathological link with CTEPH is less clear. We describe a case-series of three patients with CTEPH and one with chronic thromboembolic disease without evidence of pulmonary hypertension diagnosed following administration of high-dose EO for gender re-assignment surgery (GRAS). This constitutes a longterm component of treatment, always commenced in males preoperatively. ${ }^{3}$ 